\title{
Opportunities and potential applications from healthcare technologies to assist motor activity in metabolic myopathies
}

\author{
Ricci G, Simoncini C, Baldanzi $S$ and Siciliano $G^{*}$ \\ Department of Clinical and Experimental Medicine, Unit of Neurology, University of Pisa, Pisa, Italy
}

\begin{abstract}
The metabolic myopathies are a group of hereditary muscle disorders, characterized by a failure in the biochemical pathways of storage, mobilization and utilization of the substrates useful for energy generation and contraction of muscle cells. These diseases are classified, based on the biochemical defects, into disorders of glycogen, lipid or purine metabolism. The main symptoms due to energy deficiency are exercise intolerance, muscle cramps and myalgias, and muscle weakness caused by structural damages of muscle. Based on the exercise-related symptoms, patients with metabolic myopathies should avoid physical activities and motor training. However, it is well-known that a reduced motility causes metabolic adaptations, that can be unfavorable for these diseases. Benefits of exercise training has been documented in muscle glycogenosis, including McArdle and Pompe diseases. In this review, we also highlight some cues of new healthcare technologies opportunity to assist motor activity in disease setting, such as metabolic myopathies.
\end{abstract}

\section{Introduction}

The metabolic myopathies are a group of hereditary muscle disorders, characterized by a failure in the biochemical pathways of storage, mobilization and utilization of the substrates useful for energy generation and contraction of muscle cells [1]. These diseases are classified, based on the biochemical defects, into disorders of glycogen, lipid or purine metabolism. The exact incidence and prevalence of metabolic myopathies is uncertain. They are rare diseases, however, an improving of diagnostic capabilities in the last years have resulted in an increased number of metabolic myopathies diagnosis. Metabolic myopathies show a wide variability in term of age at onset and clinical phenotype. The majority of patients, however, present an early onset, in infancy, childhood, or young adulthood [1,2]. Main symptoms related to energy deficiency are exercise intolerance, muscle cramps and pain, exercise-induced rhabdomyolysis, but also static symptoms such as muscle weakness and atrophy, caused by structural damages of muscle, can be observed. Cardiac, smooth muscle, brain and/or endocrine dysfunctions can be part of the clinical picture. Yet there is no cure for the majority of these diseases [2].

\section{Classification of metabolic myopathies}

a) Glycogen storage diseases (or glycogenosis) are genetic disorders of glycogen metabolism, numbered based on the type of enzyme deficiency. These diseases are caused by: - accumulation of normalstructure glycogen molecules, - accumulation of abnormalstructure glycogen molecules, or -glycogen depletion. Enzymes that are required in glycogen synthesis (gluconeogenesis), glycogen degradation (glycogenolysis), or glucose metabolism (glycolysis) are involved [3].

b) Neutral Lipid Storage Diseases (NLSDs) are genetic disorders due to a non-lysosomal accumulation of neutral lipids in different tissues such as muscle, skin, heart, central nervous system, liver, thyroid, pancreas, and leukocytes. These conditions are subdivided in two main groups: Neutral Lipid Storage Disease with Myopathy (NLSD-M) and Neutral Lipid Storage Disease with Ichthyosis (NLSD-I) [4].

c) The most common muscle disorder of purine metabolism is the myoadenylate deaminase deficit (MAD), whose clinical spectrum can range from asymptomatic condition to mild exercise-induced myalgia. The disease is due to the impairment of the ability of muscle cells to process ATP, the major energy molecule [5]. Other metabolic disorders of purine metabolism can affect the nervous system, since some purines play a specialized role in the nervous system [6].

${ }^{\star}$ Correspondence to: Gabriele Siciliano, S. Chiara Hospital, Neurology Unit, via Roma 67, 56126 Pisa, Italy, Tel: +39 050 993046; E-mail: gabriele.siciliano@med.unipi.it

Key words: Metabolic myopathies, McArdle disease, Pompe disease, e-health technologies

\section{Special Issue: Assisted Exercise}

Ugo Carraro

Interdepartmental Center of Myology

University of Padova

Italy

Paolo Gargiulo

Inst. f. Biomed. and Neural

Engineering / Biomed Technology Centre

Reykjavik University \& Landspitali Reykjavik

Iceland

Alfredo Musumeci

Neuroscience Department

University of Padova \& Padova General Hospital

Italy

Received: March 16, 2018; Accepted: April 10, 2018; Published: April 14, 2018 


\section{Physiology in exercising muscles}

The knowledge of energy metabolism processes in exercising muscles is fundamental to study metabolic myopathies. During exercise, the working muscle needs a continuous supply of adenosine triphosphate (ATP), but the initial stores of ATP in the muscles are used up very quickly. Therefore, ATP must be regenerated [7]. Briefly, there are three major biochemical pathways that allow a supply of ATP within muscle cells and that can be summarized below:

a) Glycogen metabolism is involved in the aerobic pathway, that is essential for intermittent or submaximal muscle contractions and in the anaerobic pathway, that enables high-intensity muscular activity, when blood flow is reduced and oxygen availability is limited.

b) Lipid metabolism is crucial during sustained submaximal exercise, lasting longer than $40 \mathrm{~min}$.

c) Phosphocreatine stores are used up in the purine nucleotide cycle and are useful for very high-intensity exercise of short duration.

It is possible to recognize, based on exercise-related symptoms, if the alteration involved glucose or fat metabolic pathways. If the symptoms occur at the beginning of exercise within the first $10 \mathrm{~min}$, when the energy production depends on muscle glycogenolysis and glycolysis, it suggests a disease of glycogen metabolism. If the symptoms start during long-duration exercise at low-intensity, when the energy fuel derives from fatty acid oxidation, it points to a disease of lipid metabolism [8].

\section{Physical activity in metabolic myopathies}

Based on the exercise-related symptoms, patients with metabolic muscle diseases should avoid physical activities and motor training. However, it is well-known that a reduced motility causes metabolic adaptations, such as lower capacity of fatty acid oxidation and increased dependence on glycogen metabolism, that is unfavorable, for instance, for muscle glycogenosis. Benefits of exercise training has been studied in muscle glycogenosis, including McArdle and Pompe diseases, although in small sample size $[8,9]$, while there are not studies on the effect of muscle training in diseases of fatty acid oxidation. Other metabolic myopathies have been evaluated with different exercise testing, but formal training programs are yet not available $[8,9]$.

\section{Muscle training in Pompe disease (glycogen storage disease type II)}

Pompe disease, or glycogen storage disease type II, is an inherited autosomal recessive lysosomal storage muscle disorder caused by a deficiency of the enzyme acid alpha-glucosidase (GAA). The enzyme defect causes an accumulation of glycogen in the lysosomes, with secondary dysfunction and degeneration of muscle cells [10]. The classic form of infantile-onset Pompe disease begins within a few months of birth and is characterized by floppy infant appearance due to hypotonia, muscle weakness, hepatomegaly, breathing problems and heart defects with dilated cardiomyopathy. The late onset form is characterized by a variable age at onset, starting in childhood or adulthood, and degree of severity and is mainly associated with a progressive limb girdle and respiratory muscles weakness. Since 2006, the enzyme replacement therapy (ERT) with alglucosidase alfa has been available, improving life expectancy in affected children and determining a disease course stabilization with motor and pulmonary improvements in adult patients [11].

The role of exercise in Pompe disease has not been extensively studied. A certain amount of muscle activity can be supposed to be beneficial in preventing disuse atrophy and maintaining residual strength and functional status. Therefore, muscle exercise, such as aerobic and resistance training, could have a therapeutic potency in adult patients with Pompe disease.

In 2011, the group of Terzis et al. [12] studied the effect of aerobic and resistance training in adult patients with Pompe disease. They selected 5 subjects that followed twenty-week aerobic and progressive resistance exercise training. The authors observed a significant increase in muscular strength and 6-minute walking distance after training, thus suggesting the positive and save effect of muscle exercise [12]. More recently, the randomized study of exercise training, including 23 subjects affected by Pompe disease that completed a combined 12-week strength and aerobic training program, with three weekly sessions [13], showed a small but significant improvements in primary outcomes, including endurance, muscle strength and muscle function. Considering the potential benefits of exercise in Pompe disease, a recent work has also investigated if there is an alteration of the muscle substrates used during exercise. The study was performed in 7 adult patients with Pompe disease, in which the peak oxidative capacity, and carbohydrate and fatty acid metabolism during submaximal exercise for 1 hour with cycle-ergometer were tested. The main observation of this work was that patients with late-onset Pompe disease have a markedly impaired maximal aerobic exercise capacity, but their endurance is normal, since they resulted able to tolerate prolonged submaximal exercise [14].

Studies of exercise effect in infantile Pompe disease has not yet been reported. Functional submaximal exercises are generally recommended, such as cycling or walking on a treadmill [15].

\section{Muscle training in McArdle disease ((glycogen storage disease type $\mathrm{V}$ )}

McArdle's disease is the most common metabolic myopathy of muscle carbohydrate metabolism, due to deficiency of myophosphorylase and alteration of glycogen breakdown in muscle. The clinical symptoms of McArdle's disease usually begin in young adulthood with exercise intolerance, exercise-induced muscle cramps, pain and recurrent episodes of myoglobinuria [2,16]. Many patients experience the second wind phenomenon, characterized by an improved tolerance for aerobic exercise approximately after eight minutes of motor activity, secondary to the increased availability of blood glucose and free fatty acids associated to an enhanced glucose uptake by muscle cells [16]. Muscle training can have beneficial effects in patients with McArdle disease, since reduced mobility determines metabolic adaptations including a lower capacity of fatty acid oxidation and an increased utilization of glycogen metabolism, that is unfavorable in this disease. In fact, regular physical activity can improve clinical symptoms in patients with myophosphorylase deficiency [17].

Mainly three studies, involving a small number of patients, evaluated the effect of aerobic exercise training in McArdle disease. Haller and coworkers in 2006 [18] studied 8 adult patients that followed a motor training protocol on cycle ergometer for 30 to 40 minutes a day, 4 days a week, for 14 weeks, at an intensity corresponding from 60 to $70 \%$ of maximal heart rate. Their results showed that this moderate aerobic exercise protocol could be effective in improving exercise capacity. Similarly, Mate-Munoz and coworkers in 2007 [19] observed in 9 patients before and after an 8-month supervised aerobic exercise training program (including five weekly sessions of walking and/ or cycling exercise with a duration no greater than 60 minutes). The trained patients improved peak power output, peak oxygen uptake and ventilatory threshold. 
More recently (2014), Santalla and coworkers [20] investigated the effect of low-load strength training in McArdle disease. The au thors analyzed the effects of a 4-month supervised resistance (weight lifting) training program followed by a 2-month detraining period in 7 adult McArdle patients. This training program resulted safe and allowed to obtain a clear gain in muscle strength.

\section{E-Health strategies and technologies in monitoring exercise activities and its possible clinical applications}

Effect of motor training and physical activities in patients with metabolic myopathies has not been studied systematically but could be a supportive therapeutic planning. Feasible strategies to implement support for a healthy lifestyle are auspicable [21]. E-health technologies have been recognized as high-potential tools in enhancing healthcare quality, accessibility and delivery [21, 22]. Assistive and monitoring technologies can help to manage and follow up patients with these diseases. For instance, recent advances have created a market for wearable devices that measure physiological variables and bodily movement over prolonged time periods. With these purposes, in our previous work performed in collaboration with bioengineers, we designed a smart and unobtrusive techniques, able to objectively measure fatigue phenomenon and useful in monitoring muscle function in patients with a muscle diseases or in subjects with secondary skeletal muscle involvement, such as in elderly. We assessed muscle fatigue during exercise by biomedical sensors of surface electromyography using a wireless and ergonomic home-based platform [23]. Another example of mobile device recording daily life physiological parameters is the case of the SenseWear armband (SWA), that measures energy expenditure by combining accelerometry with measurements of heat production and skin conductivity [24]. By using the SenseWear armband, we defined a multi-parametric protocol in patients with McArdle's disease to detect and quantify the impairment of muscular metabolism and motor performance [16]. In this study, including 5 McArdle patients, we monitored physical activity with the electronic armband and investigated cardiopulmonary, metabolic and respiratory responses to exercise with a cardiopulmonary exercise test, also analyzing muscle fatigue during exercise test by surface electromyography. Armband monitoring of physical activity confirmed low levels of physical activity in McArdle's subjects and an impairment of both anaerobic and oxidative metabolism. Another opportunity for health monitoring derives from the smart technologie, that can provide innovative solutions for diseases management. Numerous health-related apps have been now available on the market [25]. We also recently designed an app, AIGkit, specifically conceived for adult patients with Pompe disease (article in press). AIGkit contains a section called "Motor training plan" that has been thought $s$ to create training sections mixing different exercises, taking into account the need to personalize the motor training program.

\section{Conclusion}

In conclusion, this review has highlighted some cues of new healthcare technologies opportunity and set of research challenges for promising clinical application in disease setting, such as metabolic myopathies.

\section{Author contribution}

GR and GS were equally involved in drafting of the manuscript.

\section{Declarations of interest}

The authors have no competing interest and nothing to declare.

\section{References}

1. Lilleker JB, Keh YS, Roncaroli F, Sharma R, Roberts M. Metabolic myopathies: a practical approach. Pract Neurol. 2018 Feb;18(1):14-26.

2. Angelini C1 (2015) Spectrum of metabolic myopathies. Biochim Biophys Acta 1852: 615-621. [Crossref]

3. Gazzerro E, Andreu AL, Bruno C (2013) Neuromuscular disorders of glycogen metabolism. Curr Neurol Neurosci Rep.13:333.

4. Pennisi EM, Arca M, Bertini E, Bruno C, Cassandrini D, et al. (2017) Neutral Lipid Storage Diseases: clinical/genetic features and natural history in a large cohort of Italian patients. Orphanet J Rare Dis. 12:90. [Crossref]

5. Lim L, Palayer M, Bruneau A, Letournel F, Le Maréchal C, et al. (2017) Myoadenylate deaminase deficiency: a frequent cause of muscle pain A case detected by exercise testing. Ann Biol Clin (Paris). 75:445-49. [Crossref]

6. Jinnah HA, Sabina RL, Van Den Berghe G (2013) Metabolic disorders of purine metabolism affecting the nervous system. Handb Clin Neurol 113: 1827-1836. [Crossref]

7. Volpi L, Ricci G, Orsucci D, Alessi R, Bertolucci F, et al. (2011) Metabolic myopathies: functional evaluation by different exercise testing approaches. Musculoskelet Surg 95: 59-67. [Crossref]

8. Ørngreen MC, Vissing J (2017) Treatment Opportunities in Patients With Metabolic Myopathies. Curr Treat Options Neurol. 19:37. [Crossref]

9. Preisler N, Haller RG, Vissing J (2015) Exercise in muscle glycogen storage diseases. $J$ Inherit Metab Dis 38: 551-563. [Crossref]

10. Ferreira CR, Gahl WA (2017) Lysosomal storage diseases. Transl Sci Rare Dis 2: 1-71. [Crossref]

11. van der Ploeg AT, Kruijshaar ME, Toscano A, Laforêt P, Angelini C, et al. (2017) European consensus for starting and stopping enzyme replacement therapy in adult patients with Pompe disease: a 10-year experience. Eur J Neurol 24: 768-768e31. [Crossref]

12. Terzis G, Dimopoulos F, Papadimas GK, Papadopoulos C, Spengos K, et al. (2011) Effect of aerobic and resistance exercise training on late-onset Pompe disease patients receiving enzyme replacement therapy. Mol Genet Metab. 104:279-83. [Crossref]

13. van den Berg LE, Favejee MM, Wens SC, Kruijshaar ME, Praet SF, et al. (2015) Safety and efficacy of exercise training in adults with Pompe disease: evaluation of endurance, muscle strength and core stability before and after a 12 week training program. Orphanet J Rare Dis. 10:87. [Crossref]

14. Preisler N, Laforêt P, Madsen KL, Husu E, Vissing CR, et al. (2017) Skeletal muscle metabolism during prolonged exercise in Pompe disease. Endocr Connect. 6:384-94. [Crossref]

15. Case LE, Beckemeyer AA, Kishnani PS (2012) Infantile Pompe disease on ERT: update on clinical presentation, musculoskeletal management, and exercise considerations. $\mathrm{Am}$ JMed Genet C Semin Med Genet 160C: 69-79. [Crossref]

16. Ricci G, Bertolucci F, Logerfo A, Simoncini C, Papi R, et al. (2015) A multi-parametric protocol to study exercise intolerance in McArdle's disease. Acta Myol 34: 120-125. [Crossref]

17. Ollivier K, Hogrel JY, Gomez-Merino D, Romero NB, Laforêt P, et al. (2005) Exercise tolerance and daily life in McArdle's disease. Muscle Nerve. 31:637-41. [Crossref]

18. Haller RG, Wyrick P, Taivassalo T, Vissing J (2006) Aerobic conditioning: an effective therapy in McArdle's disease. Ann Neurol 59: 922-928. [Crossref]

19. Mate-Munoz JL, Moran M, Pérez M, Chamorro-Viña C, Gómez-Gallego F, et al (2007) Favorable responses to acute and chronic exercise in McArdle patients. Clin J Sport Med. 17:297-303. [Crossref]

20. Santalla A, Santalla A, Munguía-Izquierdo D, Brea-Alejo L, Pagola-Aldazábal I, et al (2014) Feasibility of resistance training in adult McArdle patients: clinical outcomes and muscle strength and mass benefits. Front Aging Neurosci. 6:334. [Crossref]

21. Ross J, Stevenson F, Lau R, Murray E (2016) Factors that influence the implementation of e-health: a systematic review of systematic reviews (an update). Implement Sci. 11:146. [Crossref]

22. Currie WL, Seddon JJ (2014) A cross-national analysis of eHealth in the European Union: some policy and research directions. Inf Manage. 51:783-97.

23. Tartarisco G, Billeci L, Ricci G, Volpi L, Pioggia G, (2012) A personal monitoring architecture to detect muscular fatigue in elderly. Neuromuscul Disord. 22 Suppl 3:S192-197. [Crossref] 
Ricci G (2018) Opportunities and potential applications from healthcare technologies to assist motor activity in metabolic myopathies

24. Koehler K, Drenowatz C (2017) Monitoring Energy Expenditure Using a MultiSensor Device-Applications and Limitations of the SenseWear Armband in Athletic Populations. Front Physiol. 8:983. [Crossref]
25. Bonn SE, Alexandrou C, Hjörleifsdottir Steiner K, Wiklander K, Östenson CG, et al. (2018) App-technology to increase physical activity among patients with diabetes type 2 - the DiaCert-study, a randomized controlled trial. BMC Public Health. 18:119. [Crossref]

Copyright: (C2018 Ricci G. This is an open-access article distributed under the terms of the Creative Commons Attribution License, which permits unrestricted use, distribution, and reproduction in any medium, provided the original author and source are credited. 